\title{
AUDITORIA DE UM SERVIÇO DE ATENDIMENTO DE GESTANTES PORTADORAS DE DIABETES MELLITUS GESTACIONAL
}

\section{Larissa Cano de Oliveira}

Acadêmica de Medicina na Universidade da Região de Joinville - UNIVILLE, Joinville, SC, Brasil.

\section{João Pedro de Paula Bertoli}

Acadêmico de Medicina na Pontifícia Universidade $\mathrm{Ca}$ tólica do Paraná - PUCPR, Curitiba, PR, Brasil.

\section{Rodrigo Ribeiro e Silva}

Acadêmico de Medicina na Universidade da Região de Joinville - UNIVILLE, Joinville, SC, Brasil

Thiago Ribeiro e Silva

Acadêmico de Medicina na Universidade Positivo - UP, Curitiba, PR, Brasil.

\section{Matheus Leite Ramos de Souza}

Acadêmico de Medicina na Universidade da Região de Joinville - UNIVILLE, Joinville, SC, Brasil.

Jean Carl Silva

Doutor em Ciências Médicas pela Universidade Federal de São Paulo. Obstetra da Maternidade Darcy Vargas; Docente Adjunto da Universidade da Região de Joinville - UNIVILLE, Joinville, SC, Brasil.
Autor correspondente: Larissa Cano de Oliveira laricdo@gmail.com
RESUMO: Avaliar o tratamento utilizado e a classificação de peso de recém-nascidos (RN) de gestantes portadoras de Diabetes Mellitus Gestacional (DMG). Foi realizado um estudo transversal descritivo com gestantes portadoras de DMG, em gestação única, no período de abril de 2011 a fevereiro de 2016. Os dados avaliados foram o tipo de tratamento utilizado e a classificação de peso do RN. Foram avaliadas 893 gestantes. A dieta e a atividade física como única terapêutica foram utilizadas por 306 (34,3\%), 366 (41\%) necessitaram metformina, a associação de metformina e insulina foi necessária em 109 (12,2\%) e utilizaram somente insulinoterapia $112(12,5 \%)$ gestantes. Houve 27 (3,0\%) RN pequenos para a idade gestacional (PIG), 687 (76,9\%) RN adequados para a idade gestacional (AIG) e 179 (20,0\%) RN grandes para a idade gestacional (GIG). A metformina foi a terapêutica mais utilizada e a maioria dos recém-nascidos foram AIG.

PALAVRAS-CHAVES: Auditoria médica, Diabetes gestacional, Atenção terciária à saúde.

\section{AN AUDIT OF A HEALTHCARE SERVICE FOR PREGNANT WOMAN WITH GESTATIONAL DIABETES MELLITUS (GDM)}

ABSTRACT: Evaluate the treatment used and the weight classification of newborns (NB) of pregnant women with Gestational Diabetes Mellitus (GDM). A descriptive cross-sectional study was developed with pregnant women with GDM in a single gestation from April, 2011 to February, 2016. The data evaluated were the type of treatment used and the weight classification of the newborn. A total of 893 pregnant women were evaluated. Dietary and physical activity as sole therapy was used by $306(34,3 \%), 366$ (41\%) needed metformin, the combination of metformin and insulin was necessary in 109 (12,2\%) and only used insulin therapy (12,5\%) pregnant women. There were 27 (3,0\%) small for gestational age NB (SGA), $687(76,9 \%)$ adequate for gestational age NB (AGA) and $179(20,0 \%)$ large for gestational age NB (LGA). Metformin was the most used therapy and most of the newborns were AIG.

KEY WORDS: Medical Audit; Diabetes, Gestational; Tertiary Healthcare.

\section{INTRODUÇÃO}

Os diferentes tipos de Diabetes Mellitus (DM) - DM tipo I, DM tipo II e DM gestacional (DMG) - constituem um conjunto de distúrbios metabólicos caracterizados por hiperglicemia resultante de uma deficiência insulínica ${ }^{1}$. Na gestação, são considerados, juntos, a 
alteração metabólica mais frequente, com prevalência entre 3 e $25 \%$, dependendo do grupo étnico, da população e do critério diagnóstico utilizado ${ }^{2}$. Nas últimas décadas, o número de gestantes diagnosticadas com diabetes tem aumentado progressivamente em função do crescimento populacional, do aumento da idade materna, da falta de atividade física e do aumento da prevalência de obesidade - condição causadora de resistência à insulina ${ }^{1,3}$.

O DMG consiste na intolerância a carboidratos de gravidade variável, que se inicia durante a gestação atual e não preenche os critérios diagnósticos de DM franco, e é o tipo de diabetes mais frequente nesse período ${ }^{2}$. Para o diagnóstico de DMG, a Sociedade Brasileira de Diabetes recomenda que sejam seguidos os critérios propostos pela International Association of Diabetes in Pregnancy Study Group (IADPSG) e aceitos pela Organização Mundial da Saúde (OMS).

O DMG é um fator de risco independente para complicações obstétricas como parto pré-termo, préeclâmpsia, recém-nascidos (RN) grandes para a idade gestacional (GIG) e macrossômicos (peso maior do que 4 kg na gestação a termo) ou restrição de crescimento intrauterino (RCIU), traumas durante o parto, hipoglicemia neonatal e maior necessidade de cesariana ${ }^{3}$.

Indica-se o controle da hiperglicemia da gestante a fim de evitar tais complicações materno-fetais pelo diabetes na gestação. Quando as modificações do estilo de vida, como dieta apropriada associada à atividade física, não são suficientes para atingir níveis adequados de glicose sanguínea, é necessária terapia farmacológica³ A metformina pode ser usada como um agente hipoglicemiante oral seguro e efetivo ${ }^{4}$. No entanto, uma proporção de mulheres não alcança um controle glicêmico adequado com metformina isoladamente 3 . A terapia com insulina é eficaz para o controle de glicose, mas é caro e inconveniente uma vez que requer manipulação, armazenamento e refrigeração adequados ${ }^{4}$.

A falta de conhecimento sobre o Diabetes Mellitus Gestacional afeta o entendimento da gestante sobre as consequências da doença sobre o binômio mãefilho, bem como pode impactar a gestação em sí. É de extrema importância o monitoramento dos desfechos obstétricos das metodologias de tratamento utilizadas para que correções possam ser realizadas e os resultados melhorados. O presente estudo tem como objetivo avaliar o tratamento utilizado e a classificação de peso de recém-nascidos (RN) de gestantes portadoras de DMG. Como desfechos secundários, foram avaliados o perfil epidemiológico das gestantes com DMG de um serviço de atendimento terciário e os resultados materno-fetais mais relacionados ao diabetes na gestação.

\section{METODOLOGIA}

Foi realizado um estudo transversal descritivo que traçou o perfil epidemiológico, tratamento e desfechos materno-fetais de todas as gestantes portadoras de DMG, atendidas durante o período de abril de 2011 a fevereiro de 2016, e que tiveram o parto realizado na Maternidade Darcy Vargas (MDV) - Joinville, SC.

Todas as gestantes incluídas, em gestação única e que realizaram acompanhamento pré-natal por diabetes na gestação no serviço de Alto Risco da MDV, preencheram os critérios diagnósticos para DMG segundo as diretrizes da Sociedade Brasileira de Diabetes, propostos pela International Association of Diabetes in Pregnancy Study Group (IADPSG). No primeiro trimestre, em idade gestacional (IG) menor do que 20 semanas, a glicemia de jejum da gestante com valor entre 92 e $125 \mathrm{mg} / \mathrm{dL}$ indica diagnóstico de DMG; caso ultrapasse $126 \mathrm{mg} / \mathrm{dL}$, sugere diagnóstico de DM prévio. No segundo trimestre, entre 24 e 28 semanas de gestação, é realizado o teste de Tolerância Oral à Glicose (TTOG) 75g. Os valores de referência para glicemia de jejum são de 92 a $125 \mathrm{mg} / \mathrm{dL}$, glicemia após $1 \mathrm{~h} \geq 180 \mathrm{mg} / \mathrm{dL}$ ou após 2h de 153 a 199 $\mathrm{mg} / \mathrm{dL}$, sendo que um ponto alterado na curva já faz o diagnóstico de $\mathrm{DMG}^{6}$. Foram excluídas as gestantes com dados incompletos no prontuário.

Foi utilizada como terapêutica a metodologia do escore clínico-laboratorial descrito por do Valle et al. ${ }^{7}$. No trabalho, o escore final foi calculado pela soma dos valores, entre -2 e +2 , de cada medida considerada: glicemia em jejum, glicemia pós-prandial, circunferência abdominal fetal, índice de massa corporal (IMC) e IG no momento da consulta. As recomendações para o tratamento do diabetes durante a gestação foram baseadas no escore final, cujo intervalo no qual o resultado se encontra sugere nova consulta com nutricionista, manutenção 
da dieta e atividade física, hipoglicemiante oral, insulina concomitante à dieta e/ou hipoglicemiante oral.

Todos os dados foram retirados do Prontuário Único do Paciente (PUP) da MDV. Os dados maternos coletados para análise foram idade, número de gestações anteriores, IMC, ganho de peso na gestação, IG de início de tratamento, doença hipertensiva específica da gestação (DHEG) na gestação e hipertensão arterial sistêmica (HAS) anterior à gestação. Os resultados relacionados ao diabetes incluíram resultado de TTOG, tipo de tratamento utilizado - dieta, metformina, insulina ou metformina + insulina -, glicemia média em jejum e pósprandial durante o pré-natal e hemoglobina glicosilada (HbA1c) no terceiro trimestre. Os resultados coletados relacionados aos recém-nascidos foram IG do parto, prematuridade ( $I G<37$ semanas), via de parto, peso ao nascer de acordo com a curva de crescimento intraútero, desenvolvida por Lubchenco et al. ${ }^{8}$, Apgar, malformações, necessidade de UTI e óbito.

Os dados quantitativos foram processados pelo cálculo de médias e desvios-padrão. Para as variáveis nominais, foram calculadas as frequências absolutas e relativas.

A MDV assinou a Declaração de Exequibilidade do Projeto de Pesquisa, ao pesquisador principal, docente do Departamento de Medicina e do Programa de Mestrado / Doutorado em Saúde e Meio Ambiente da Universidade da Região de Joinville - Univille, para realizar a coleta de dados no prontuário das gestantes. Este projeto foi aprovado pelo Comitê de Ética em Pesquisa (CEP) do Hospital Regional Hans Dieter Schmidt, Joinville, SC, Brasil, sob CAAE de número 82477318.1.000.5363.

\section{RESULTADOS}

Foram avaliadas 893 gestantes. A média de idade das gestantes atendidas foi de 30,76 anos, com 2,71 gestações anteriores. A análise do IMC de acordo com a classificação definida pela OMS constatou que 39,3\% eram obesas. A IG de início de tratamento do DMG foi de 28,41 semanas. $O$ percentual das mulheres que apresentaram DHEG é $6,5 \%$ e $13,1 \%$ eram portadoras de HAS anterior à gestação. As características maternas analisadas estão compiladas na Tabela 1.
Tabela 1. Características maternas*

\begin{tabular}{|ll|}
\hline Idade & $\mathbf{3 0 , 7 6}(6,35)$ \\
\hline Gestações anteriores & $2,71(1,70)$ \\
\hline IMC & $29,12(6,12)$ \\
\hline Classificação do IMC & \\
\multicolumn{1}{|c|}{ Baixo peso } & $69(7,7 \%)$ \\
\hline \multicolumn{1}{|c|}{ Peso adequado } & $204(22,8 \%)$ \\
\hline \multicolumn{1}{|c|}{ Sobrepeso } & $250(28,0 \%)$ \\
\hline \multicolumn{1}{|c|}{ Obesa } & $351(39,3 \%)$ \\
\hline Ganho de peso & $4,51(6,56)$ \\
\hline IG de início de tratamento & $28,41(6,88)$ \\
\hline DHEG na gestação & $58(6,5 \%)$ \\
\hline HAS anterior à gestação & $117(13,1 \%)$ \\
\hline
\end{tabular}

Fonte: Autores, 2019.

*Média e desvio-padrão, números absolutos e percentagens; IMC: Índice de Massa Corporal; IG: Idade Gestacional; DHEG: Doença Hipertensiva Específica da Gestação; HAS: Hipertensão Arterial Sistêmica

Em relação ao diabetes (Tabela 2), a média do TTOG de jejum foi de 93,16 mg/dL, 171,47 mg/dL após 1 hora e 150,16 mg/dL após 2 horas. O valor médio de HbA1c foi de $5,40 \%$. A glicemia média de jejum foi de $89,39 \mathrm{mg} / \mathrm{dL}$ e a pós-prandial de 117,18 mg/dL durante o tratamento. Dentre os tratamentos utilizados pelas gestantes, a monoterapia com metformina foi o mais prevalente $(41,0 \%)$.

Tabela 2. Resultados relacionados ao diabetes*

\begin{tabular}{|ll}
\hline Teste de Tolerância Oral à Glicose (TTOG) & \\
\hline Jejum & $93,16(13,37)$ \\
\hline $\mathbf{1}$ hora & $171,47(33,28)$ \\
\hline $\mathbf{2}$ horas & $150,26(30,42)$ \\
\hline Tipo de tratamento utilizado & \\
\hline Dieta & $306(34,3 \%)$ \\
\hline Metformina & $366(41,0 \%)$ \\
\hline Insulina & $112(12,5 \%)$ \\
\hline Metformina + Insulina & $109(12,2 \%)$ \\
\hline Glicemia média & \\
\hline Jejum & $89,39(12,72)$ \\
\hline Pós-prandial & $117,18(16,92)$ \\
\hline HbA1c & $5,40(0,47)$ \\
\hline
\end{tabular}

Fonte: Autores, 2019.

*Média e desvio-padrão, números absolutos e percentagens; HbA1c: Hemoglobina Glicosilada

A IG média do parto foi de 38,52 semanas. Neste estudo, 6,5\% dos bebês nasceram pré-termo. A via de parto foi cesariana em $52,9 \%$ das gestantes. O peso médio 
dos recém-nascidos foi de 3.324,69 gramas, sendo que $20,0 \%$ dos neonatos foram classificados como GIG, e 6,7\% eram macrossômicos. Em relação ao Apgar, 6,4\% e 1,5\% dos recém-nascidos apresentaram Apgar baixo $(<7)$ no $1^{\circ}$ e $5^{\circ}$ minuto, respectivamente; $5,7 \%$ necessitaram de UTI e $0,6 \%$ foram a óbito. Os demais dados relacionados aos desfechos neonatais encontram-se na Tabela 3.

Tabela 3. Resultados relacionados ao recém-nascido*

\begin{tabular}{|c|c|}
\hline Idade gestacional do parto & $38,52(1,45)$ \\
\hline Prematuridade & $58(6,5 \%)$ \\
\hline \multicolumn{2}{|l|}{ Via de parto } \\
\hline Parto normal & $421(47,1 \%)$ \\
\hline Cesariana & $472(52,9 \%)$ \\
\hline Peso do recém-nascido & $3324,69(515,66)$ \\
\hline \multicolumn{2}{|l|}{ Classificação de peso do RN } \\
\hline PIG & $27(3,0 \%)$ \\
\hline AIG & $687(76,9 \%)$ \\
\hline GIG & $179(20,0 \%)$ \\
\hline Macrossômico (> $4 \mathrm{Kg})$ & $60(6,7 \%)$ \\
\hline \multicolumn{2}{|l|}{ Apgar } \\
\hline $1^{\circ}$ minuto & $8,02(1,21)$ \\
\hline Baixo $1^{\circ}$ minuto & $57(6,4 \%)$ \\
\hline $5^{\circ}$ minuto & $8,96(0,94)$ \\
\hline Baixo $5^{\circ}$ minuto & $13(1,5 \%)$ \\
\hline Necessidade de UTI & $51(5,7 \%)$ \\
\hline Óbito & $5(0,6 \%)$ \\
\hline Malformação & $31(3,5 \%)$ \\
\hline \multicolumn{2}{|c|}{$\begin{array}{l}\text { Fonte: Autores, } 2019 . \\
\text { *Média e desvio-padrão, números absolutos e percentagens; PIG } \\
\text { Pequeno para a Idade Gestacional; AIG: Adequado para a Idade } \\
\text { Gestacional; GIG: Grande para a Idade Gestacional; UTI: Unidade } \\
\text { de Terapia Intensiva }\end{array}$} \\
\hline
\end{tabular}

\section{DISCUSSÃO}

A auditoria do serviço de atendimento de gestantes portadoras de DMG da MDV revelou que o perfil dessas foi equivalente ao encontrado por outros estudos semelhantes. A terapêutica mais utilizada para o controle da glicemia materna foi a metformina, tal como sugere a literatura, porém houve algumas diferenças em relação aos desfechos neonatais.

A análise do perfil da população estudada verificou que a média de idade foi de 30,76 anos, o que vai de acordo com o estudo de Massucatti, Pereira e Maioli 9 , ao sugerir que mulheres na faixa etária de 20 a 40 anos apresentam maior prevalência de diabetes gestacional.
Não foi encontrada correlação entre o número de gestações anteriores e o DMG, porém, em uma análise de prevalência ${ }^{9}$, observou-se que $56,6 \%$ apresentaram três ou mais gestações, enquanto a média neste trabalho foi de 2,71 gestações prévias.

A inadequação do estado nutricional das gestantes pode favorecer intercorrências na gravidez como diabetes e pré-eclâmpsia, e seu controle é relevante visto que se trata de um fator de risco modificável ${ }^{10}$. Autores evidenciaram que o risco de DMG é diretamente proporcional ao IMC materno ${ }^{11}$, e, no trabalho de Simon, Marques e Farhat ${ }^{12}$, é referido caso em que houve verificação de maior prevalência de DMG em gestantes com sobrepeso ou obesidade, tal como visto no presente trabalho. Entretanto, outros estudos ${ }^{10,13}$ mostram que pode ocorrer de a maioria das gestantes diabéticas encontrar-se no peso adequado.

Uma vez que quanto mais precoce o início do controle da hiperglicemia na gestação, melhores tendem a ser os desfechos materno-fetais; espera-se que o tratamento de DMG seja iniciado a partir da primeira consulta no ambulatório de gestação de alto risco. A IG média do diagnóstico de DMG no estudo de Carocha et $a l .{ }^{14}$ foi de 27 semanas. Na população estudada, o início do tratamento da hiperglicemia materna, independente da terapia escolhida, foi de 28,41 semanas. SimeonovaKrstevska et al..$^{15}$ analisaram separadamente as gestantes que iniciaram o tratamento com dieta, metformina ou insulina, e encontrou que a IG média foi de 29,5, 28,6 e 24 semanas nos respectivos grupos.

A glicemia de jejum das gestantes pesquisadas foi de $89,39 \mathrm{mg} / \mathrm{dL}$ e a pós-prandial foi de $117,18 \mathrm{mg} / \mathrm{dL}$, valores correspondentes aos de outro estudo ${ }^{16}(16)$, que, no entanto, também considerava glicemia de jejum $\geq 92$ $\mathrm{mg} / \mathrm{dL}$ como um dos possíveis critérios para o diagnóstico que DMG.

Nesta auditoria, 13,1\% das gestantes apresentavam HAS anterior à gestação, ligeiramente abaixo de um estudo em que esse número foi de $19,8 \%{ }^{17}$ e superior a outro em que $8,7 \%$ das gestantes diabéticas eram portadoras de HAS prévia?.

Mulheres com DMG têm um risco de 10 a 30\% de desenvolverem pré-eclâmpsia ${ }^{16}$. Neste estudo, 6,5\% das gestantes com DMG desenvolveram DHEG, o que se 
compara àquelas com tolerância normal à glicose, cujo risco é de 5 a $7 \%$. Já no estudo de Aktün et al. ${ }^{18}$, apenas $3 \%$ das gestantes com DMG desenvolveram pré-eclâmpsia, enquanto no de Filho et al. ${ }^{19}$, essa taxa foi de $19,4 \%$. Apesar da diferença significativa entre os resultados encontrados por esses autores e de que a metformina não tenha sido objeto dos estudos, sabe-se que o seu uso está associado à redução do risco para $\mathrm{DHEG}^{20}$.

A HbA1c foi mostrada como um fator importante para o aumento da incidência de $\mathrm{RN} \mathrm{GIG}^{15}$, mas não há consenso sobre um valor de referência a partir do qual esse risco seja modificado. Encontrou-se um valor de $\mathrm{HbA} 1 \mathrm{c}$ de 5,40\% entre as gestantes analisadas, semelhante ao encontrado por Weinert et al. ${ }^{21}$, que foi de 5,9\%. Os resultados de Renz (2018) ${ }^{22}$ mostraram que, a partir do ponto de corte de 5,8\% para HbA1c, tem-se especificidade suficiente para o diagnóstico do DMG. Em ambos os casos, conforme esperado, a HbA1c de mulheres com DMG foi mais baixa do que os valores previstos para mulheres com DM prévio $(\geq 6,5 \%)^{23}$.

A primeira intervenção visando ao controle da hiperglicemia materna consiste em orientação alimentar. Neste estudo, 34,3\% das gestantes diabéticas efetuaram controle glicêmico apenas com dietoterapia, enquanto na população de Neta $e t$ al. ${ }^{24}$, uma porcentagem bastante superior de gestantes $(78,0 \%)$ obteve sucesso apenas com tratamento não medicamentoso. Se após duas semanas de dieta os níveis glicêmicos permanecerem elevados, devese iniciar tratamento farmacológico ${ }^{6}$, o qual é necessário para $20 \%$ a $60 \%$ das pacientes com $\mathrm{DMG}^{25}$. Porém, encontrou-se que $65,7 \%$ das gestantes necessitaram de terapia com metformina, insulina ou da associação de ambas.

Durante muito tempo, a insulina foi usada como tratamento padrão para o DMG. Entretanto, a segurança de hipoglicemiantes orais como a metformina tem sido demonstrada quando somente a dieta não é suficiente para atingir níveis glicêmicos desejados ${ }^{26}$. Alguns benefícios do tratamento inicial com metfomina em relação à insulina incluem menor número de prematuridade e partos cesáreos, redução do ganho de peso materno e de desfechos neonatais desfavoráveis como macrossomia e admissão em serviços de cuidados neonatais especiais ${ }^{26}$.
A taxa de sucesso na terapêutica com a metformina nas gestantes portadoras de DMG em ensaios clínicos randomizados varia entre 53 e $75 \%^{27}$. Outro estudo $^{25}$ apontou que $46,3 \%$ dos pacientes tratados com metformina necessitaram de insulina suplementar. $\mathrm{Na}$ população analisada, a monoterapia com metformina foi o tratamento mais prevalente, eficaz em $41,0 \%$ das gestantes, o que diminuiu a necessidade da associação de insulina à terapêutica, que foi necessária para 12,2\% das pacientes que faziam uso de metformina. Quando as metas glicêmicas maternas não forem atingidas com a dose máxima de metformina, a terapêutica é substituída pela insulinoterapia. Alguns autores acreditam que até 60\% das mulheres com DMG necessitam de tratamento com insulina ${ }^{4}$. Neste estudo, $12,5 \%$ das gestantes diabéticas utilizaram insulina em monoterapia, porém essa porcentagem é reduzida quando a metformina é utilizada. No estudo de Amaral et al. ${ }^{13}$, a insulinoterapia foi o tratamento mais prevalente, utilizado por $36,1 \%$ das gestantes.

No presente estudo, a idade gestacional do parto foi de 38,52 semanas. Houve $6,5 \%$ de prematuridade entre os casos analisados, o que vai de encontro com outro estudo ${ }^{26}$, o qual aborda que a taxa de bebês prétermos na literatura varia de $4 \%$ a $16 \%$. Porém esses resultados contrastaram com o resultado obtido por outros trabalhos semelhantes, em que a incidência de partos prematuros foi de $16,7 \%{ }^{19}$ e de $25,5 \%{ }^{28}$.

Alguns estudos apontam incidência maior de cesárea nas gestantes diabéticas provavelmente pela maior incidência de macrossomia feta ${ }^{28}$. As indicações de parto cesáreo para gestantes diabéticas podem aumentar pelo maior risco de essas gerarem um filho $\mathrm{GIG}^{26}$. Nesta auditoria, a cesariana foi via de parto em $52,9 \%$ dos casos, o que supera a de outros estudos (13,28), em que 32,9\% e $37,2 \%$ dos partos foram por essa via. Já no estudo de Filho et al. ${ }^{19}$ houve $100 \%$ de partos cirúrgicos, porém a população analisada era pequena. No trabalho de Silva, Rangel e Reis ${ }^{17}, 91,6 \%$ tiveram parto cesariano.

Macrossomia fetal é observada em até 30\% dos casos e pode predispor a traumas durante $o$ parto ${ }^{16}$. Entretanto, neste estudo, a incidência de fetos macrossômicos foi de $6,7 \%$, bastante abaixo do que a referência anterior, porém acima de outros resultados 
encontrados na literatura ${ }^{18,26}$, nos quais essa taxa não ultrapassou $2 \%$ entre filhos de mães com DMG. Encontraram-se 20,0\% de neonatos GIG e 3,0\% PIG. Aktün et al. ${ }^{18}$ encontraram a mesma proporção de recémnascidos PIG, porém a proporção de GIG encontrada foi de $9 \%$, o que contrasta com a desta auditoria. A literatura relata que os AIGs oscilam em torno de $86,6 \%{ }^{18}$, na população estudada encontrou-se $76,9 \%$, o que pode ser resultado de um diagnóstico mais precoce do DMG ou do ajuste na terapêutica realizado no serviço analisado.

$\mathrm{O}$ índice de Apgar fornece um método aceito e conveniente para relatar o status do recém-nascido imediatamente após o nascimento ${ }^{29}$. Encontrou-se que 6,4\% dos recém-nascidos apresentaram Apgar baixo no primeiro minuto após o nascimento, enquanto outros estudos obtiveram $5,6 \%^{26}$ e $8,6 \%{ }^{13}$. Apenas $1,5 \%$ dos recém-nascidos tiveram Apgar abaixo de 7 no quinto minuto, valor próximo a $1,1 \%$ e $2,3 \%$, encontrados em outros estudos ${ }^{26,28}$.

A admissão na UTI neonatal foi necessária para 5,7\% dos recém-nascidos da população analisada, incidência semelhante à encontrada por Aktün et al. ${ }^{18}$ (6\%), porém pouco mais da metade do que a taxa de $10,5 \%$ encontrada por outro estudo ${ }^{28}$. Na literatura, a necessidade de UTI varia de $2 \%$ a $23,5 \%{ }^{26}$.

No trabalho de Moraes et al. ${ }^{30}$, que avalia a taxa de óbito fetal em um hospital referência em gestações de alto risco, a porcentagem de óbito fetal de gestantes com DMG foi de $16,7 \%$, significativamente maior do que a que foi encontrada na população pesquisada, que foi de apenas $0,6 \%$.

Os resultados relacionados ao diabetes tenderam a seguir um padrão e variaram pouco, porém sabe-se que podem sofrer alterações conforme os critérios utilizados para o diagnóstico e a terapêutica escolhida, para os quais não há um esquema único. Porém, conhecer nosso resultado é importante para que possamos comparar os desfechos obtidos com as condutas terapêuticas em nosso atendimento ao de outros serviços que acompanham gestantes diabéticas.

\section{CONCLUSÃO}

O perfil das gestantes com DMG atendidas pelo serviço de Alto Risco da Maternidade Darcy Vargas é bastante semelhante ao de outros centros. Nota-se que foi utilizada mais metformina que outros serviços, o que pouco interferiu na maioria dos desfechos, que foram de acordo com os encontrados na literatura. Contudo, destaca-se que a porcentagem de GIG no serviço pesquisado foi maior do que a encontrada por outros estudos, e a taxa de óbitos fetais foi mais baixa.

\section{REFERÊNCIAS}

1. Organização Pan-Americana da Saúde; Ministério da Saúde; Federação Brasileira das Associações de Ginecologia e Obstetrícia; Sociedade Brasileira de Diabetes. Rastreamento e Diagnóstico de Diabetes Mellitus Gestacional no Brasil. Brasília, DF: OPAS; 2017.

2. Sociedade Brasileira de Diabetes (SBD). Diretrizes da Sociedade Brasileira de Diabetes 2017-2018. São Paulo: SBD; 2017.

3. Gante I, Melo L, Dores J, Ruas L, Almeida M do C. Metformin in gestational diabetes mellitus: predictors of poor response. European Journal of Endocrinology. 2018;178(1):129-35.

4. Silva JC, Pacheco C, Bizato J, de Souza BV, Ribeiro TE, Bertini AM. Metformin compared with glyburide for the management of gestational diabetes. International Journal of Gynecology \& Obstetrics. 2010;111(1):37-40.

5. Karsten LF, de Souza DL, Vieira MR, Silva JC. Influência do diagnóstico de Diabetes Mellitus Gestacional na qualidade de vida da gestante. Saúde e Pesquisa. 2016; 9(1):7-14.

6. Sociedade Brasileira de Diabetes (SBD). VI Diretrizes da Sociedade Brasileira de Diabetes 20152016. São Paulo: SBD; 2016.

7. Valle JB do, Silva JC, Oliveira DS, Martins L, Lewandowski A, Horst W. Use of a clinicallaboratory score to guide treatment of gestational diabetes. International Journal of Gynecology \& Obstetrics. 2018;140(1):47-52.

8. Lubchenco LO, Hansman C, Dressler M, Boyd E. 
Intrauterine growth as estimated from liveborn birth-weight data at 24 to 42 weeks of gestation. Pediatrics. 1963;32(5):793-800.

9. Massucatti LA, Pereira RA, Maioli TU. Prevalência de Diabetes Gestacional em Unidades de Saúde Básica. Revista de Enfermagem e Atenção à Saúde. 2012;01(01):70-9.

10. Oliveira ACM de, Graciliano NG. Síndrome hipertensiva da gravidez e diabetes mellitus gestacional em uma maternidade pública de uma capital do Nordeste brasileiro, 2013: prevalência e fatores associados. Epidemiologia e Serviços Saúde. 2015;24(3):441-51.

11. Bolognani CV, Souza SS de, Calderon I de MP. Diabetes mellitus gestacional - enfoque nos novos critérios diagnósticos. Comunicação em Ciências da Saúde. 2011;22(Supl 1):31-42.

12. Simon CY, Marques MCC, Farhat HL. Glicemia de jejum do primeiro trimestre e fatores de risco de gestantes com diagnóstico de diabetes melito gestacional. Revista Brasileira de Ginecologia e Obstetrícia. 2013;35(11):511-5.

13. Amaral AR, Silva JC, Ferreira BDS, E Silva MR, Bertini AM. Impacto do diabetes gestacional nos desfechos neonatais: uma coorte retrospectiva. Scientia Medica. 2015;25(1):19272.

14. Carocha A, Rijo C, Amaral N, Aleixo F, Rocha T. Diabetes Gestacional e Hemoglobina A1c. Revista Portuguesa de Endocrinologia, Diabetes e Metabolismo. 2012;01:15-20

15. Simeonova-Krstevska S, Bogoev M, Bogoeva K, Zisovska E, Samardziski I, Velkoska-Nakova V, et al. Maternal and Neonatal Outcomes in Pregnant Women with Gestational Diabetes Mellitus Treated with Diet, Metformin or Insulin. Macedonian Journal of Medical Sciences. 2018;6(5):803-7.

16. Abi-Abib RC, Cabizuca CA, Carneiro JRI, Braga FO, Cobas RA, Gomes MB, et al. Diabetes na gestação. Revista Hospital Universitário Pedro Ernesto. 2014;13(3):40-7.

17. Silva $C$, Rangel $C$, Reis A. Prevalência e análise clínico-epidemiológica do diabetes mellitus gestacional no município de Campos dos Goytacazes, RJ. Revista Científica da FMC. 2016;11:12-4

18. Aktün LH, Uyan D, Yorgunlar B, Acet M. Gestational diabetes mellitus screening and outcomes. Journal of the Turkish German Gynecological Association. 11 de março de 2015;16(1):25-9.

19. Filho CRC, Leite JJG, Mesquita CAM, de Araujo TMLA, de Sousa UMC, Silva MJL, Silva MJL et al. Características clínicas e epidemiológicas de recém-nascidos e mães diabéticas. Conexão Ciência (Online). 2017;12(2):47-53.

20. Romero R, Erez O, Hüttemann M, Maymon E, Panaitescu B, Conde-Agudelo A, et al. Metformin, the aspirin of the 21 st century: its role in gestational diabetes mellitus, prevention of preeclampsia and cancer, and the promotion of longevity. American Journal of Obstetrics and Gynecology. 2017;217(3):282-302.

21. Weinert LS, Oppermann MLR, Salazar CC, Simionato BM, Silveiro SP, Reichelt AJ. Diabetes e gestação: perfil clínico e laboratorial em pré-natal de alto risco. Revista HCPA. 2010;30(4):334-41.

22. Renz PB. Hemoglobina glicada (HbA1c) no diabetes mellitus gestacional. Porto Alegre. Tese [ Doutorado em Ciencias médicas : endocrinologia ] - Faculdade de Medicina da UFRGS; 2018.

23. Universidade Federal do Rio Grande do Sul (UFRS) [homepage na internet]. Diabetes Mellitus. [acesso em 21 jan. 2019]. Disponível em: https://www.ufrgs.br/telessauders/documentos/ protocolos_resumos/endocrino_resumo_diabetes TSRS_20160324.pdf

24. Neta FAV, Crisóstomo VL, Castro RCMB, Pessoa SMF, Aragão MMS, Calou CGP. Avaliação do perfil e dos cuidados no pré-natal de mulheres com diabetes mellitus gestacional. Revista da Rede Enfermagem do Nordeste. 2014;15(5):823-31.

25. Priya G, Kalra S. Metformin in the management of diabetes during pregnancy and lactation. Drugs in Context. 2018;7:1-21.

26. Silva AL da, Amaral AR do, Oliveira DS de, Martins L, Silva MR e, Silva JC. Desfechos neonatais de acordo com diferentes terapêuticas do diabetes mellitus gestacional. Jornal de Pediatria. 2017;93(1):87-93.

27. Silva JC, Souza BV de, Silva MR e. Preditores de sucesso da metformina no tratamento do diabetes mellitus gestacional. Revista Brasileira de Saúde Materno Infantil. 2013;13(2):129-35.

28. Zanrosso CD, Schuch T, Camassola M, Pizzolotto 
L, Bisotto C, Andreazza T, et al. Desfechos materno-fetais do diabetes gestacional em serviço terciário de atenção obstétrica. Revista da AMRIGS. 2013;59(2):112-5

29. American Academy of Pediatrics Committee on Fetus and Newborn; American College of Obstetricians and Gynecologist Committee on Obstetric Practice. The apgar score. Pediatrics. 136 (4); 2015

30. Silva MMB, Shiguematsu I, Póvoa DMM, Ikeda A, Ferraro LS, Souza IMD de, et al. Fatores associados ao óbito fetal em um hospital de referência em gestação de alto risco. Revista UNILUS Ensino e Pesquisa. 2017;14(36):68-80.

Recebido em: 29/01/2019

Aceito em: 28/08/2019 